\title{
Integrated long-range thermal bimorph actuators for parallelizable bio-AFM applications
}

\author{
Jonas Henriksson, Maurizio Gullo and Juergen Brugger, Senior member, IEEE
}

\begin{abstract}
AFM-based cell force spectroscopy is an emerging research method that already has enhanced our understanding of the structural changes that take place in a cell as it becomes cancerous. However, the method is limited as it is not timeefficient in its current state of development. This paper presents the fabrication of an integrated long-range thermal bimorph actuator that controls the z-position of an AFM cantilever in liquid. Multiplied in arrays, such individually actuated probes can parallelize cell force spectroscopy measurements, thereby drastically reducing the time per measured cell. The need to accommodate differences in tip-sample distance implies an individual device actuation range of $\geq 10 \mu \mathrm{m}$ out-of-plane. In addition, any cross-talk, i.e. between actuators or between the actuator and the force sensor, must be minimized. To meet these requirements, we designed and fabricated a novel thermal bimorph actuator that was paired with a force sensing cantilever. In order to keep temperatures in a bio-friendly range, the design was optimized for high thermomechanical sensitivity. FEM simulations confirmed that the surrounding liquid constitutes a large thermal reservoir that absorbs the generated heat without any dramatic temperature increase. Furthermore, given that a cell substrate material of high thermal conductivity is chosen, e.g. $\mathrm{Si}$, the thermal coupling between the cell and the substrate dominates over that between the cell and the actuator. Suspended silicon nitride structures with platinum electrodes were microfabricated through standard techniques. The finalized actuator was able to displace the cantilever out-of-plane by $\sim 17 \mu \mathrm{m}$ in air, corresponding well to estimations.
\end{abstract}

Index Terms-Atomic Force Microscopy, Force sensors, Microactuators, Microsensors

\section{INTRODUCTION}

$\mathrm{I}^{\mathrm{n}}$ $\mathrm{n}$ recent years, there has been a surge of research focusing on the role of the biomechanical properties of cells in the onset and progression of human diseases [1]. For example, it has been observed that the cancerous state of a cell is coupled with significant changes of its Young's modulus and adhesion

Manuscript received February 1, 2013. This work was supported financially by the Nano-Tera Swiss federal program through the PATLiSci project. (Corresponding author: Jonas Henriksson)

An earlier version of this paper was presented at the $11^{\text {th }}$ IEEE Sensors Conference in 2012 (Taipei, Taiwan) and was published in its proceedings.

The authors are with the Microsystems Laboratory, EPFL, Lausanne, $\mathrm{CH}-$ 1015, Switzerland (phone: +41 21 6936573; fax: +41 21 6936670; e-mail: juergen.brugger@epfl.ch, jonas.henriksson@epfl.ch).

Copyright (c) 2012 IEEE. Personal use of this material is permitted. However, permission to use this material for any other purposes must be obtained from the IEEE by sending a request to pubs-permissions@ieee.org. force. This fact is believed to be crucial in understanding the process of metastasis formation. To measure the mechanical properties of biological samples, the atomic force microscope (AFM) has emerged as an appropriate metrology tool due to its capability to provide high force sensitivity while working in an aqueous environment [2]-[6].

At the time of its invention in 1986, the AFM was built for scanning of inorganic surfaces, providing sub-nanometer vertical resolution in air [7]. The next major event was microfabrication of AFM cantilevers [8], leading to high through-put device production as well as a two orders of magnitude size reduction. This miniaturization implied an increase in resonance frequency, greatly enhancing the scan speed - a crucial development that more recently enabled realtime observation of cellular processes [9]. The applicability of AFM cantilevers is still expanding, counting magnetic actuation, free of parasitic resonance peaks, as well as heating control to its toolkit [10].

Studies have shown that AFM-based cell force spectroscopy measurements can be used to discriminate between healthy and cancerous cells, suggesting a potential basis for diagnostics [11]-[12]. However, while speed enhancement brought scanning into the biophysical domain, an equivalent leap forward in cell force spectroscopy is yet to be implemented. Admittedly, a single cell measurement may require a minimum time of a few tens of seconds. In order to get a statistically sound basis of data, thousands of sample measurements may be necessary. Not only does that mean that the collection of data is tedious and time-consuming; it also implies a general weakening of the data, as time-related factors may influence the measurements.

The reality is that as long as probing is carried out serially, cell by cell, the lengthy repetitions needed to gather a sufficient amount of data constitutes a significant bottleneck for the usage of AFM-based cell force spectroscopy. In order to circumvent this issue, a more time-efficient approach that parallelizes measurements could prove instrumental.

Several works have highlighted the increased timeefficiency that arrays of cantilevers working in parallel can bring, including piezoelectrically actuated AFM cantilevers [13], VLSI of $32 \times 32$ AFM cantilevers that can perform $\mathrm{read} /$ write/erase memory functions through thermally assisted patterning of a polymer film [14] as well as dip-pen nanolithography [15]. More specifically for biological applications, 2D cantilever arrays were developed to perform parallel scanning in liquid by Polesel-Maris et al. [16]. Favre 
et al. [17] implemented this approach to the force spectroscopy field, using an interferometric readout method in order to reduce the device level complexity. Loizeau et al. [18] refined the fabrication of such arrays, achieving high-density arrays with precise control of the spring constant and tip properties of each cantilever using a molding process. The tip mold fabrication featured iterations of oxidation and etching of a pyramidal cavity on a $\mathrm{Si}$ wafer, resulting in very high tips $(+10 \mu \mathrm{m})$ with desired tip radii $(\sim 5 \mu \mathrm{m})$.

Nevertheless, a disadvantage of passive cantilever arrays is that they cannot accommodate topographic differences between the points that are being probed in parallel, meaning that the height of each cell is not exactly the same. Moreover, such devices cannot correct eventual fabrication-related variations affecting the relative position of the tip. The result is that the ensemble of measurements is inhomogeneous in indentation depth and, consequently, in actual force applied to each cell. The only way to fully resolve this is to implement individual actuation of each cantilever in the array.

In this work, we present the design, fabrication and characterization of arrays of individually actuated force sensing cantilevers for cellular biomechanical measurements. The device was designed for compatibility with the interferometric readout method, which is described in detail elsewhere [17]. In addition to evident requirements, such as compatibility between the actuator and the surrounding media, the device must also meet:

1) A static displacement range in $\mathrm{z}$ of more than $10 \mu \mathrm{m}$ in liquid.

2) Within a few degrees of margin, the structure must remain flat. This is necessary in order to get a precise readout signal.

3) An efficient separation between force sensing and actuation, meaning that the actuation behavior is largely the same, even if forces are exerted on the cantilever by the sample.

4) The actuator should be inert to biological systems.

5) Actuators should neither cross-talk with each other, nor with the force sensors.

6) A tip of 3-5 $\mu \mathrm{m}$ in radius and $\geq 10 \mu \mathrm{m}$ in height must be fabricated at the free end of the force sensing cantilever.

There are rather few attempts at realizing liquid-compatible, actuated cantilever probes that work in parallel. Piezoelectrically actuated cantilever arrays have been reported in literature [19]; the same type of system was adapted to inliquid applications by passivation using a PDMS elastomer [20]. Akiyama et al. [21] designed a 10x10 cantilever system in which individual PZT blocks, insulated by Mylar film, provided the actuation. The advantages of the latter approach include the separation of the actuation system from the probes. However, the actuation range of piezoelectric systems is not large enough for our constraints and, moreover, it is not clear how to implement them in a simple way that fulfills the flat structure requirement.

A careful study of the available MEMS actuation techniques led to the conclusion that thermal bimorph actuation has the best chance of fulfilling all requirements.
The main challenge is to assure that the cells will not be harmed by the heat generated by the actuator. However, it is worth noting that thermal actuation has already been used for cell imaging before by Fantner et al. [22], relying on the fact that the surrounding water functions as a very large thermal reservoir that can absorb the energy dissipating from the MEMS. In our work, we investigate the potential of expanding the thermal actuation approach to long range static displacement as a means of achieving high throughput in the field of cell force spectroscopy.

In order to enhance the thermomechanical sensitivity of a bimorph-based temperature sensor, a special configuration was proposed by Lim et al. [23], named flip-over-bimorph beams (FOBs). These structures could displace out-of-plane without changing the endpoint angle, as shown in fig. 1 . The underlying principle is based on the alternating patterning of two stress-inducing layers, one on the top and one on the bottom of the structural layer. The zero angle endpoint enables the connection of several cantilevers, forming a serpentine spring, leading to enhanced thermomechanical sensitivity while keeping a small footprint. By adding a heating resistor, the structure can be made thermally actuated, shown by Jia et al. [24].
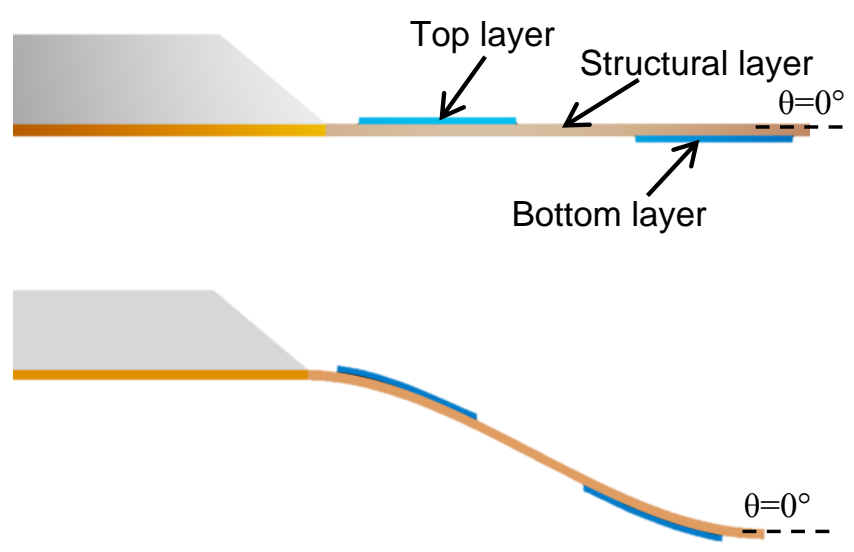

Fig. 1. A cantilever consisting of a structural layer with two stress-inducing layers of equivalent geometry, one on the top and one on the bottom. In response to a temperature change, an out-of-plane deformation will result without any change of the endpoint angle, $\theta$.

With these ideas as starting point, we designed a novel structure in which the electrode works both as a resistor that regulates the temperature and as a stress-inducing layer that causes a bending of the structure. This innovative idea means

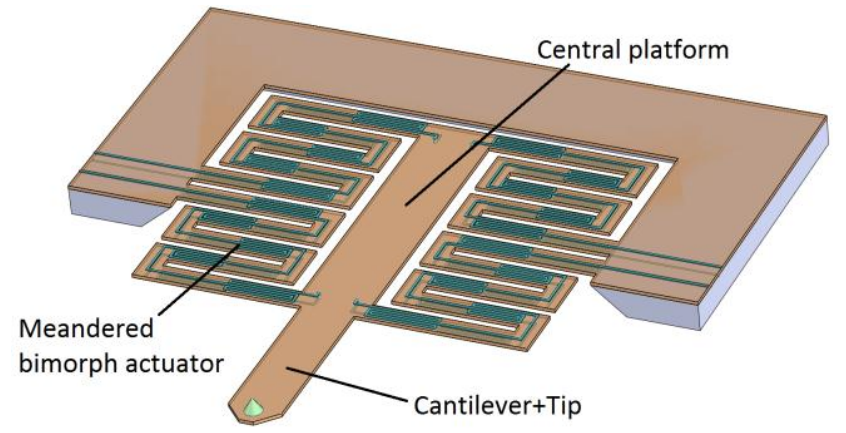

Fig. 2. Schematic of the structure. Four meandered bimorph actuator springs displace the structure in $\mathrm{z}$. Indicated is also the sensing cantilever and its tip. 
a simplification of the fabrication while maintaining a high performance. Fig. 2 shows how four such meandered bimorph actuators working as actuators connect to a central platform. If a current is applied, the actuators displace the platform away from the substrate. The platform is connected to a force sensing cantilever, equipped with a tip. By tuning the ratio between their respective spring constants, sensing and actuation can be mechanically decoupled.

To quantify the thermomechanical sensitivity of the structure, we consider a single unit beam of a serpentine spring. Its deflection can be regarded as the accumulated deflection of two identical beams of half its length, described by [23]

$$
\delta_{\text {single }}=\frac{1}{2} \kappa\left(\frac{L}{2}\right)^{2} \cdot 2=\kappa \frac{L^{2}}{4}
$$

where $L$ is the beam length and $\kappa$ is the curvature, the latter determined by [25]

$$
\kappa=\frac{M}{E I}
$$

where $E$ is the combined modulus of elasticity and $I$ is the moment of inertia. $M$ is the stress moment. As shown by Pulskamp et al. [26], a set of equations (see supplementary information) allows this property, and thereby also the vertical deflection, to be determined precisely for the case of stress actuated cantilevers. We applied the same set of equations, using the standard thermal strain equation

$$
\sigma=\alpha T
$$

where $\alpha$ is the thermal expansion coefficient and $T$ is the temperature.

Finally, the deflection of the full serpentine scales linearly with the number of meanders, $n$,

$$
\delta=\delta_{\text {single }} \cdot n
$$

The set (1)-(4) was used to determine device geometries that could fulfill the specifications.

\section{FABRICATION}

\section{A. Process flow}

As outlined in fig. 3, the process started with a double-side polished $\mathrm{Si}$ wafer (p-type, <100>) with a sputtered $\mathrm{SiO}_{2}$ layer of $1.5 \mu \mathrm{m}$ on one side (a). Using RIE, a back-side hard-mask was patterned from the $\mathrm{SiO}_{2}$ layer (b) as preparation for the last step, i.e. the through wafer etching. In order to achieve sufficient optical access, a pattern of small openings in the hard-mask was defined that, through the RIE lag effect, allowed a controlled thinning of the $\mathrm{Si}$ in proximity to the wafer's apertures during the last step. Subsequently, the Pt bottom electrodes $(200 \mathrm{~nm})$ were deposited on the front side by lift-off (c). A $1.5 \mu \mathrm{m}$ layer of silicon nitride was deposited by PECVD (d) and was subsequently patterned by RIE to form the various parts of the final structure (e). Then, a 200 $\mathrm{nm}$ Pt top electrode layer was deposited and patterned by liftoff (f). A tip made from SU-8 was patterned (g) and at last a combination of RIE and $\mathrm{KOH}$ was used to release the structure $(\mathrm{h})$

a.

b.

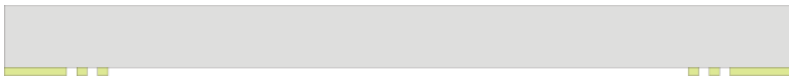

c.

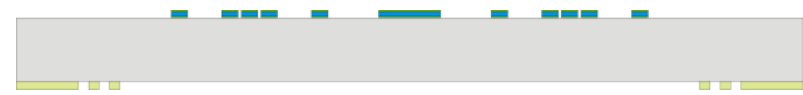

d.

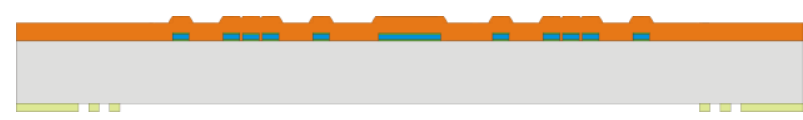

e.

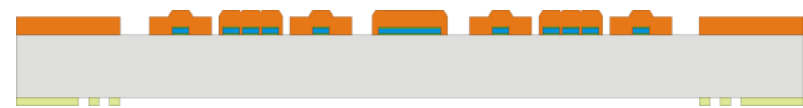

f.

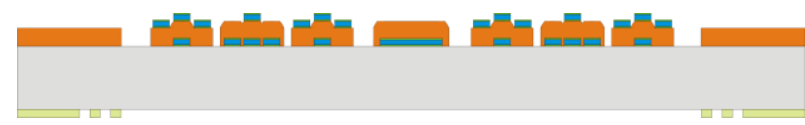

g.

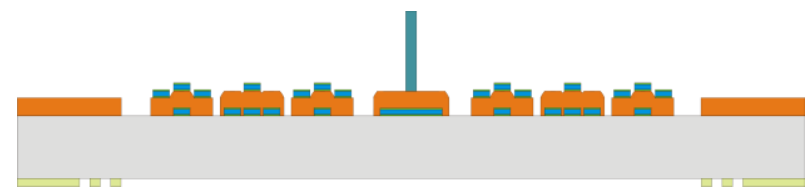

h.

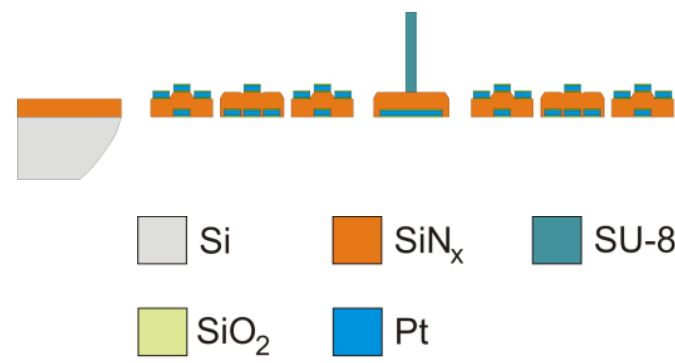

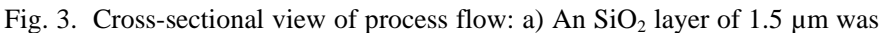
sputtered onto one side of a double-side polished $\mathrm{Si}$ wafer. b) The $\mathrm{SiO}_{2}$ layer was patterned to serve as hard-mask during the release step. c) Then, the bottom electrodes were patterned through lift-off deposition of Pt $(200 \mathrm{~nm})$. d) A $1.5 \mu \mathrm{m}$ PECVD $\operatorname{SiN}_{\mathrm{x}}$ layer was deposited and e) patterned through RIE. f) Top electrodes of Pt ( $200 \mathrm{~nm}$ ) were patterned by lift-off. g) An SU-8 tip was patterned and $h$ ) the structure was released through RIE and $\mathrm{KOH}$ etching.

The chips were designed to be suspended on cantilever beams after the etch-through step, allowing convenient release of the particular chip by breaking off its fixations when ready. Subsequently, the chip was glued to a PCB and wire-bonded. In order to passivate the electrodes, a conformal $1 \mu \mathrm{m}$ layer of parylene was deposited onto the devices. 
This article has been accepted for publication in a future issue of this journal, but has not been fully edited. Content may change prior to final publication.

Henriksson, Gullo and Brugger: Long-range thermal actuators for parallelizable bio-AFM

\section{B. RIE lag effect based Si thinning patterns}

As previously mentioned, the hard-mask was patterned so that the wafer would be thinned down close to the main apertures during the through-wafer etch process. This was possible thanks to the RIE lag effect [27]-[28], which stipulates that trenches or square apertures of exposed $\mathrm{Si}$, having a smallest dimension equal to $1-5 \mu \mathrm{m}$, will have a significantly lower etch rate than apertures larger than $10 \mu \mathrm{m}$. Fig. 4 shows a test pattern including a large opening, meant for a completed etch-through, with a surrounding pattern of small circular apertures, aimed to thin down the silicon around the main aperture. The image was taken after Bosch process etching, with the $\mathrm{SiO}_{2}$ hard-mask still present. Fig. 5 was acquired after $\mathrm{O}_{2}$ cleaning, followed by isotropic Si etching and finally removal of the hard-mask. In the design, the individual radius and density of small apertures decrease as the radial distance from the center of the main aperture increases. The image reveals that, by varying these parameters, it is possible to achieve an arbitrary thinning of the $\mathrm{Si}$ in parallel with an etch-through process.

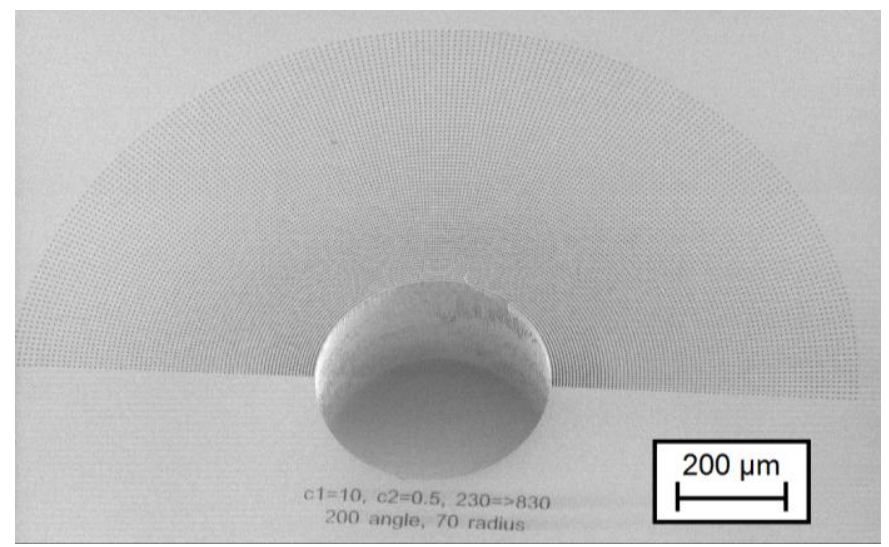

Fig. 4. SEM image acquired after Bosch process etching, transferring the test pattern of the hard-mask onto the Si substrate. The test pattern includes the main central aperture surrounded by an amphitheater of small $(\sim 3 \mu \mathrm{m})$ circular apertures.

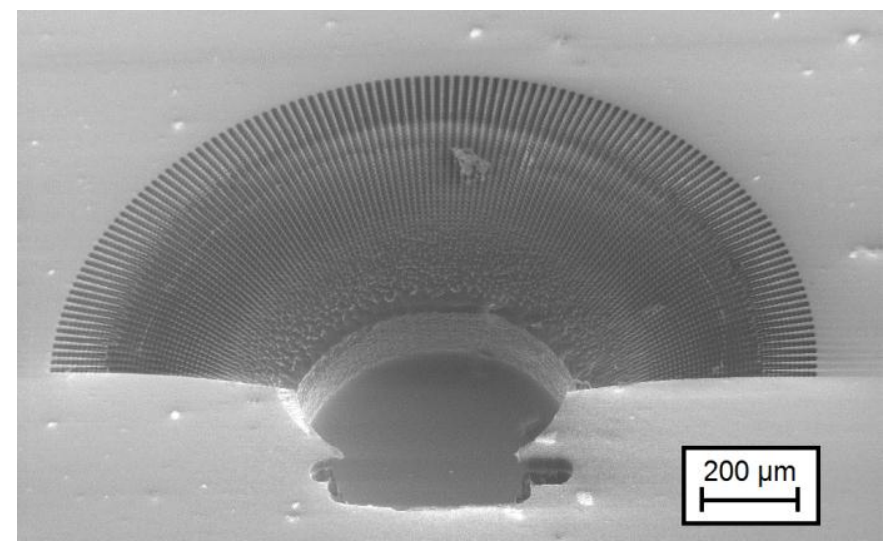

Fig. 5. SEM image after isotropic dry etching of $\mathrm{Si}$ and subsequent removal of the $\mathrm{SiO}_{2}$ hard-mask. The designed variations in size and density of the small apertures has induced a slope, indicating that higher density of apertures and/or larger aperture size implies a higher etch rate.

\section{Tip fabrication}

The tips were fabricated using Gersteltec GM1070 SU-8 negative tone epoxy. After an $\mathrm{O}_{2}$ plasma surface treatment, the resist was spun onto the wafer at about $3500 \mathrm{rpm}$, giving a final thickness of about $20 \mu \mathrm{m}$. A softbake entailed, ramping up to $130^{\circ} \mathrm{C}$, then keeping this temperature for $300 \mathrm{~s}$ and finally ramping down slowly. Given that the radial size of the tips implied 3-5 $\mu \mathrm{m}$ mask apertures, the exposure dose was increased by about $50 \%$ compared to the standard dose so that SU-8 at the interface with the underlying silicon nitride would surely become cross-linked during the post-exposure bake (PEB). During the PEB, the temperature rose to $100^{\circ} \mathrm{C}$. A relaxation time of $24 \mathrm{~h}$ followed in order to reinforce adhesion. The development in PGMEA was carried out very carefully due to concerns that the structures might easily detach. The adhesion was very critical, as such small structures have a rather short life-time in a $\mathrm{KOH}$ bath. The inclusion of an anchoring layer could improve this. A tip, before release, is shown in fig. 6 .

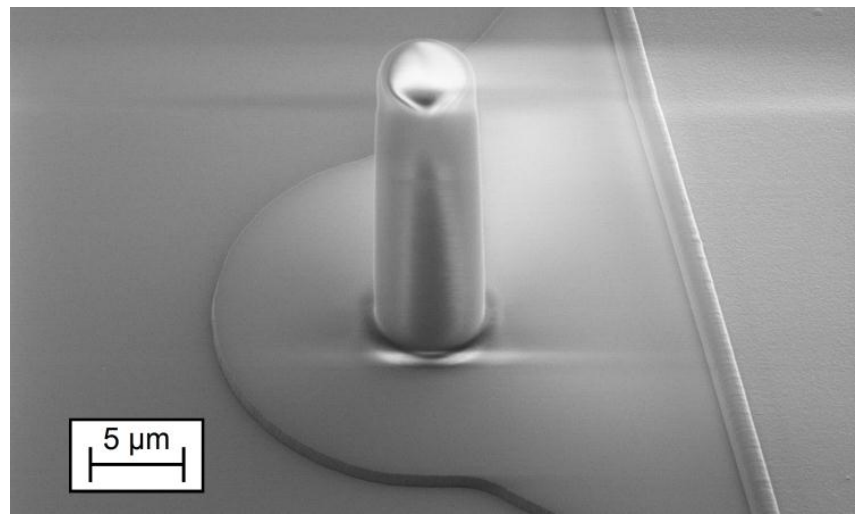

Fig. 6. SEM image of an SU-8 tip at the cantilever endpoint. The height is 20 $\mu \mathrm{m}$ and the radius is about $5 \mu \mathrm{m}$.

\section{RESULTS}

\section{A. SEM images of finalized devices}

The final release is done in $\mathrm{KOH}$, rendering a released structure as shown in fig. 7. The lower image reveals the topbottom electrode pattern alternation which is the basis for inducing a stress momentum to bend the structure in the desired direction.

An optical reference structure (ORS), shown in fig. 7, was added to the structure design in order to facilitate the readout. A soft spring connects the platform to the ORS, giving a linear, attenuated displacement of the ORS with respect to the platform. The attenuation is determined by the ratio between the spring constants of the four actuator springs and the ORS spring. In cases where the cantilever moves through a distance of several wavelengths very suddenly, the ORS is designed to resolve the problem of phase ambiguity.

To assess the optical accessibility, fig. 8 shows a SEM image of the wafer's backside aspect. The gradual opening, i.e. thinning of the $\mathrm{Si}$ in proximity to the aperture, follows the hard-mask pattern. The observed step-wise slopes and planes appeared during the $\mathrm{KOH}$ etching. 
This article has been accepted for publication in a future issue of this journal, but has not been fully edited. Content may change prior to final publication.

Henriksson, Gullo and Brugger: Long-range thermal actuators for parallelizable bio-AFM

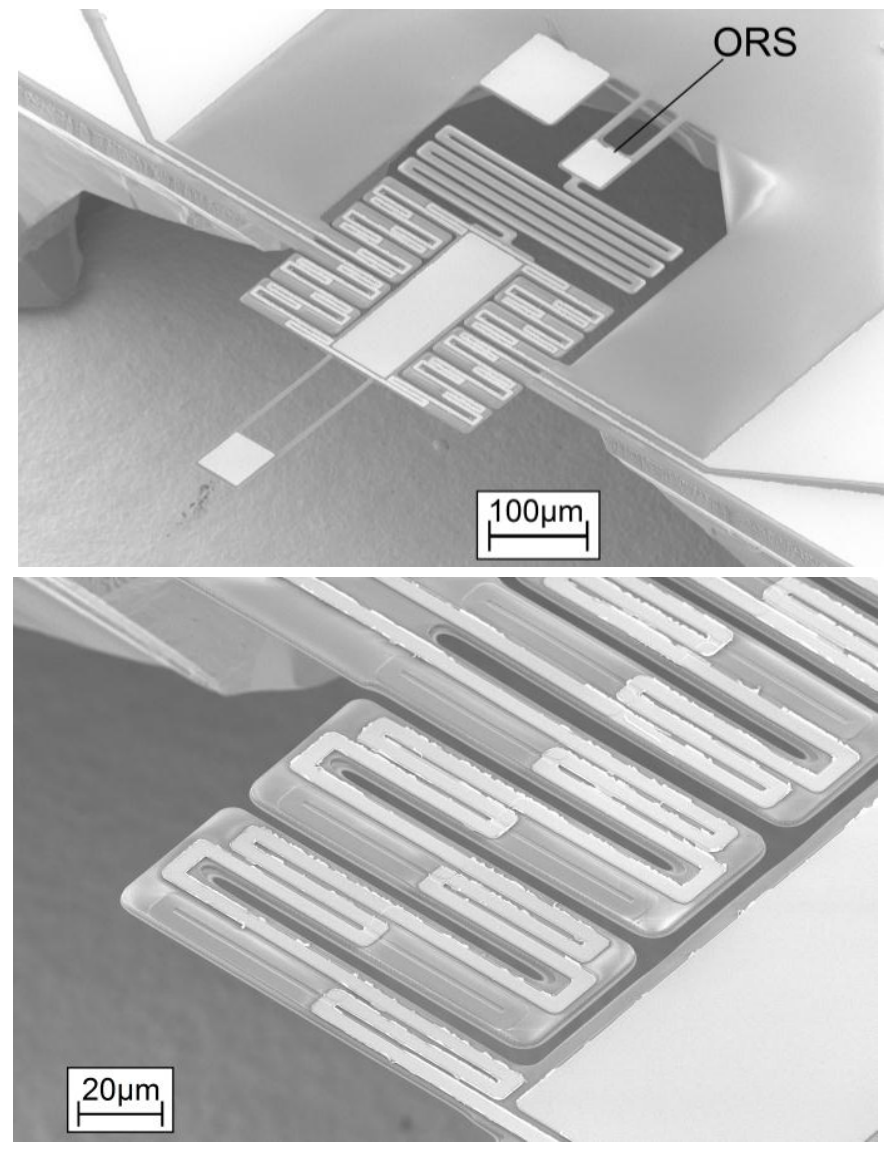

Fig. 7. SEM images of a finalized device (top) and a zoom-in (bottom) of one of its actuator springs. The latter shows more specifically the Pt electrode pattern. Some flags are also visible, due to a $20 \mathrm{~nm}$ thin Pt sputter deposition that completes an interconnection that is present on few devices. The optical reference structure (ORS) is indicated in the top image.

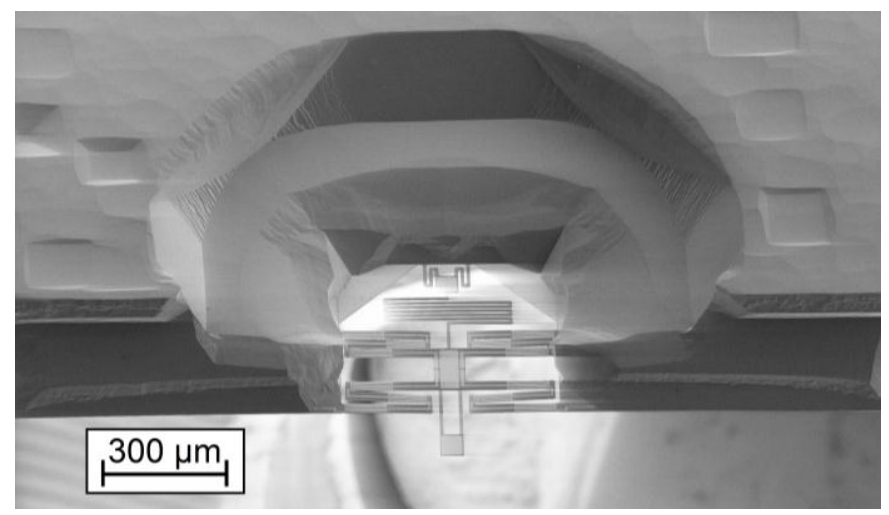

Fig. 8. A SEM image acquired from the chip's backside aspect. The shape of the opening was defined by the hard-mask. The image reveals that it is possible to engineer the shape of the opening in a highly arbitrary way by using the RIE etch lag effect.

Fig. 9 shows a $1 \times 2$ array, indicating that 1D scaling could be achieved.

\section{B. Device characterization}

The displacement range of the actuator, working in air, was measured using a Veeco Wyko NT1100 optical profiler. Simultaneously, the current passing through the electrodes was measured using a multimeter. Based on those measurements, the change of temperature was determined using the temperature coefficient of resistivity for Pt. This

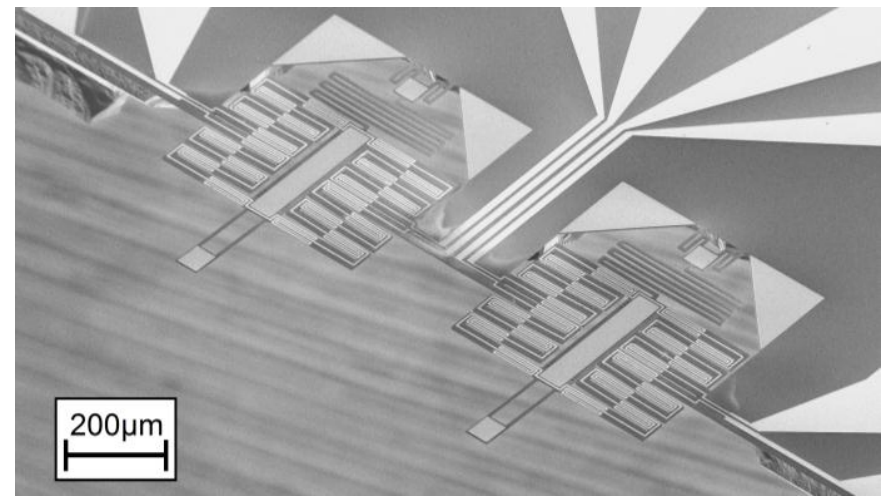

Fig. 9. A 1x2 array of devices.

coefficient was measured specifically for the samples using a hotplate (see supplementary information). The displacement and temperature results are shown in fig. 10, indicating a displacement range close to $17 \mu \mathrm{m}$ and a maximum temperature change of $212.8 \mathrm{~K}$. Fig. 11 presents the displacement plotted as function of temperature. According to equations (1)-(3), this relationship is linear in theory. Based on the data points in fig. 11 , an averaged coefficient of $\sim 77.6$ $\mathrm{nm} / \mathrm{K}$ was determined. Using the approach outlined in the introduction, an analytical estimation of the deformation is also plotted in the same figure for comparison.

Furthermore, fig. 10 also shows the ORS displacement together with the very same data points scaled up by a factor 31.2 , chosen to minimize the difference with the platform's displacement. The plot reveals that the mechanical coupling between the platform and the ORS is linear and attenuated as required.

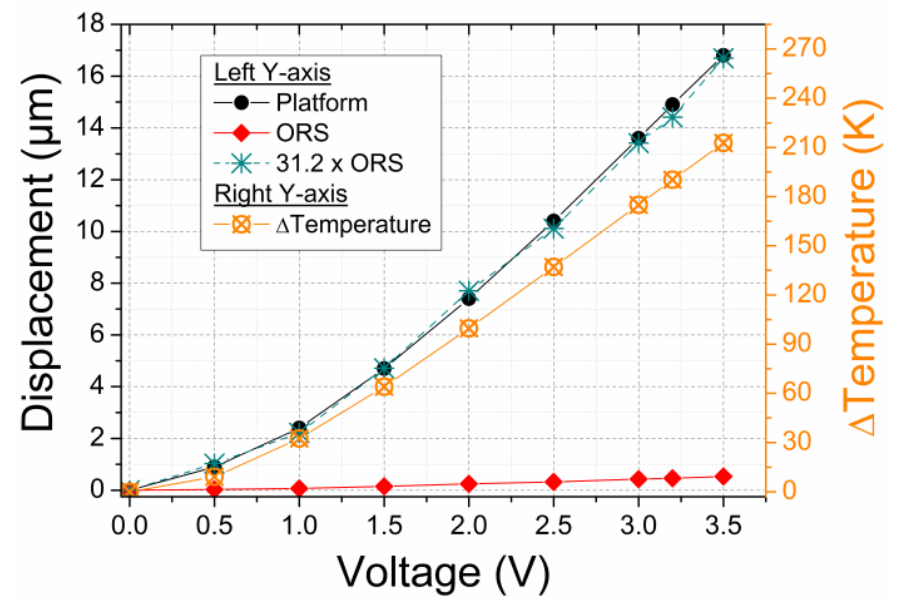

Fig. 10. Out-of-plane displacement in air in function of voltage applied across the heating resistor. A maximum platform displacement of about $16.8 \mu \mathrm{m}$ was reached. The displacement of the optical reference structure (ORS) is plotted and, to compare with the platform deformation, the very same data points are plotted again but scaled up by a factor 31.2, indicating the linear behavior of the spring connecting the ORS to the platform. The temperature of the electrodes is also plotted (orange crossed-over circles), with the Y-axis on the right side. 


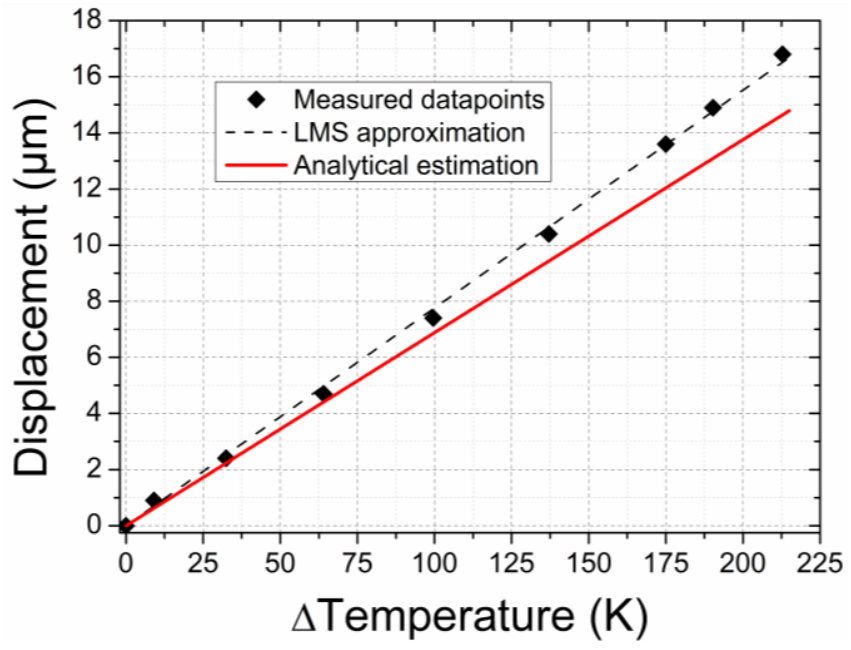

Fig. 11. Measured displacement plotted as function of electrode temperature (black diamond) together with linear LMS approximation with a coefficient of $77.6 \mathrm{~nm} / \mathrm{K}$. Also plotted in red is the result of an analytical estimation of the deformation for the particular geometry, giving a coefficient of 68.8 $\mathrm{nm} / \mathrm{K}$.

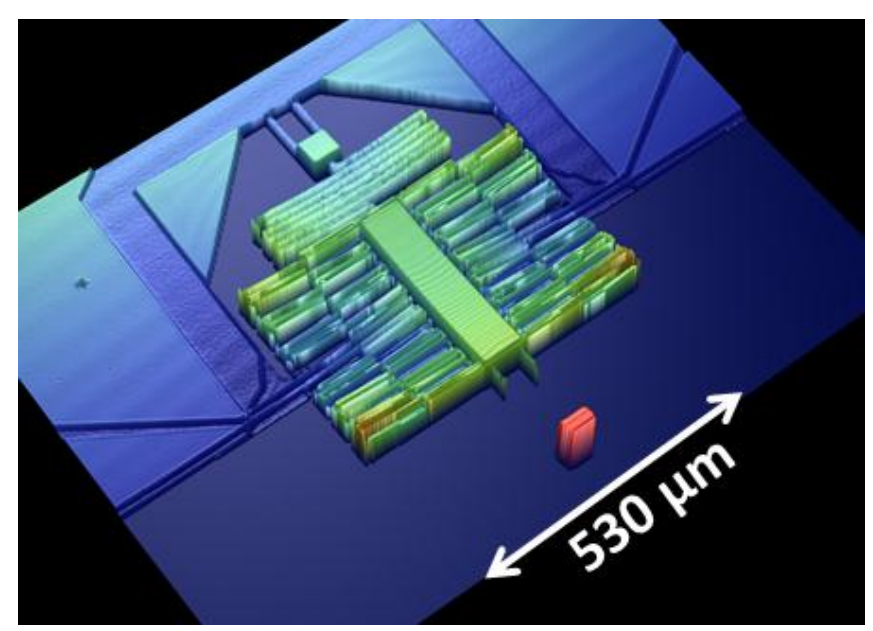

Fig. 12. Optical profilometer image of an actuated structure.

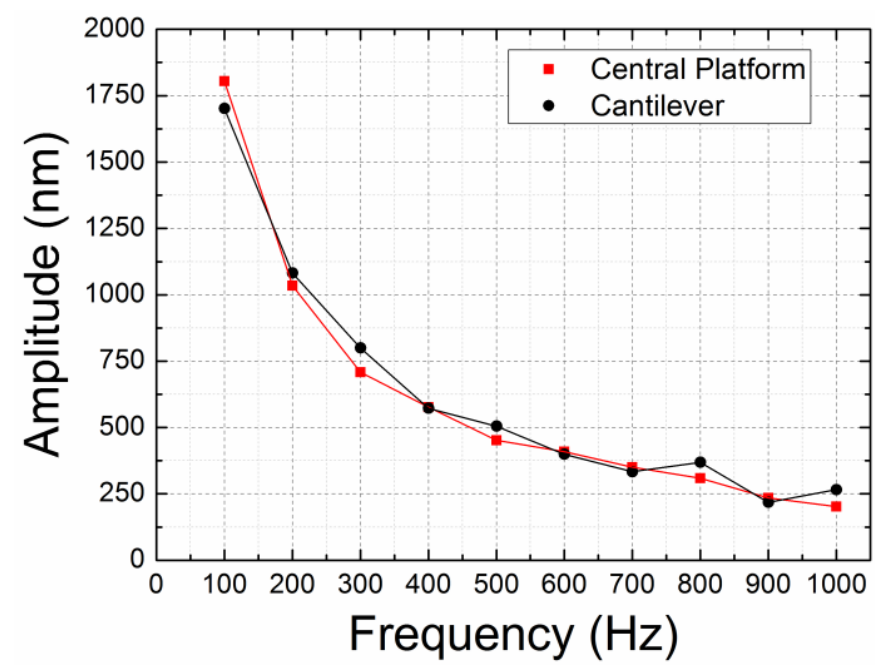

Fig. 13. The plot shows the peak-to-peak motion amplitude of a device in function of actuation frequency. The drop in amplitude as frequency increases indicates that $100 \mathrm{~Hz}$ is already beyond or around to the thermal cut-off frequency.
The actuation's frequency-dependence was assessed using a Veeco Wyko NT 1100 optical profiler with the DMEMS module, which includes a stroboscopic functionality. Fig. 12 shows the resulting 3D image of an actuated structure. The device was actuated using a square function input of $1.5 \mathrm{~V}$. The peak-to-peak motion amplitude is plotted in fig. 13 as a function of frequency, ranging from 100 to $1000 \mathrm{~Hz}$ by 100 $\mathrm{Hz}$ steps. Over this interval, the amplitude fell from $\sim 1750 \mathrm{~nm}$ down to $\sim 250 \mathrm{~nm}$ as a consequence of the thermal time constant of the system. Through simulations, it was determined that the mechanical cut-off would be at a frequency of several $\mathrm{kHz}$, depending on geometry. The falling amplitude indicates that the device did not have enough time to cool down and then heat up again during a single phase.

In the next experimental step, it was confirmed that a passivated device could be actuated in water. Experiments to determine the displacement range are currently ongoing.
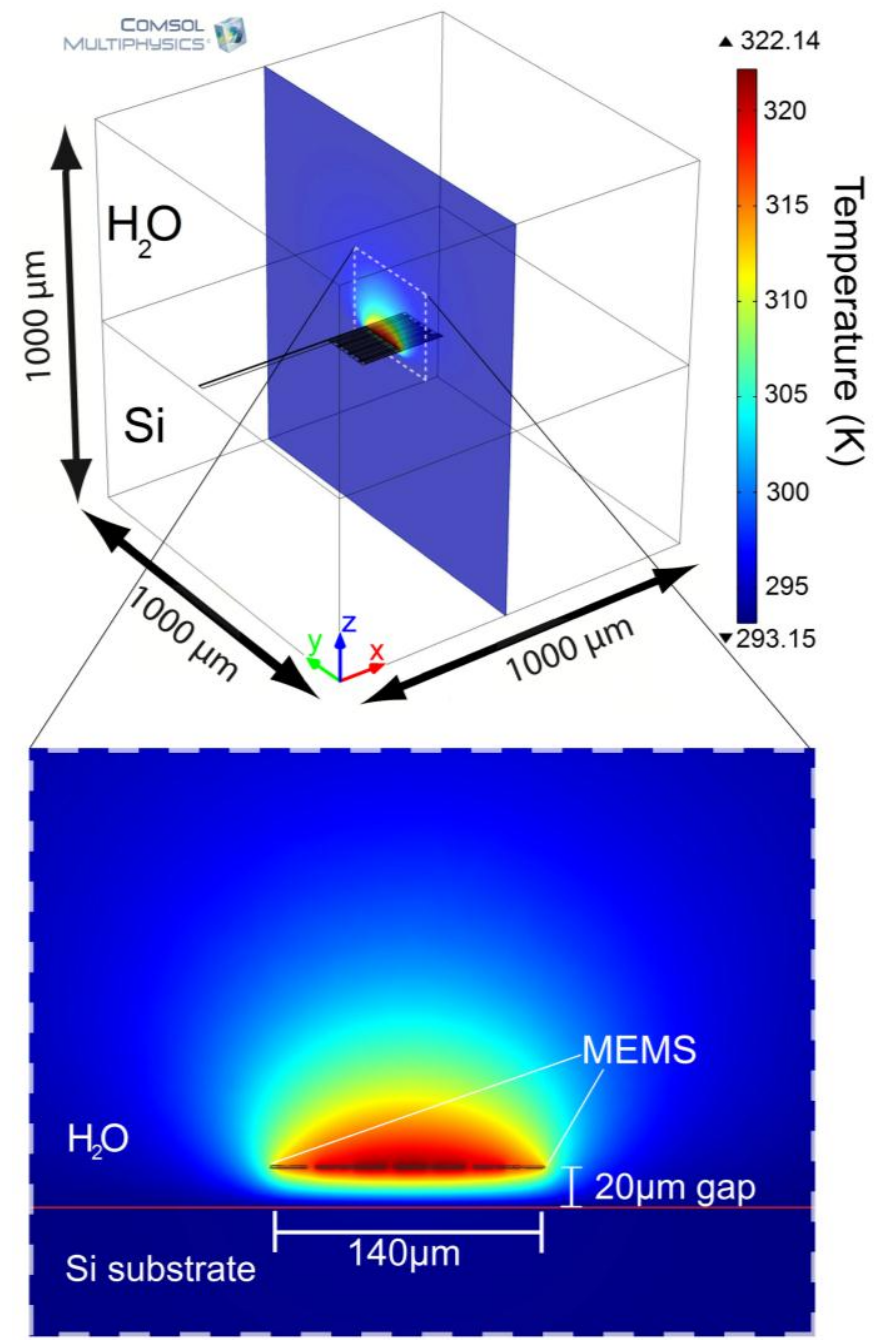

Fig. 14. Overview (top) of simulation, showing an actuator in a water volume, positioned close to the interface with a silicon substrate. The steady state solution determines the temperature throughout the volume as a result of joule heating originating from the structure's Pt heater. The yz cross-section passes through the center of the heater, illustrating the volume's temperature profile. The zoom-in (bottom) of the cross-section highlights the temperature profile close to the structure. The gap between the structure and the substrate is 20 $\mu \mathrm{m}$. 


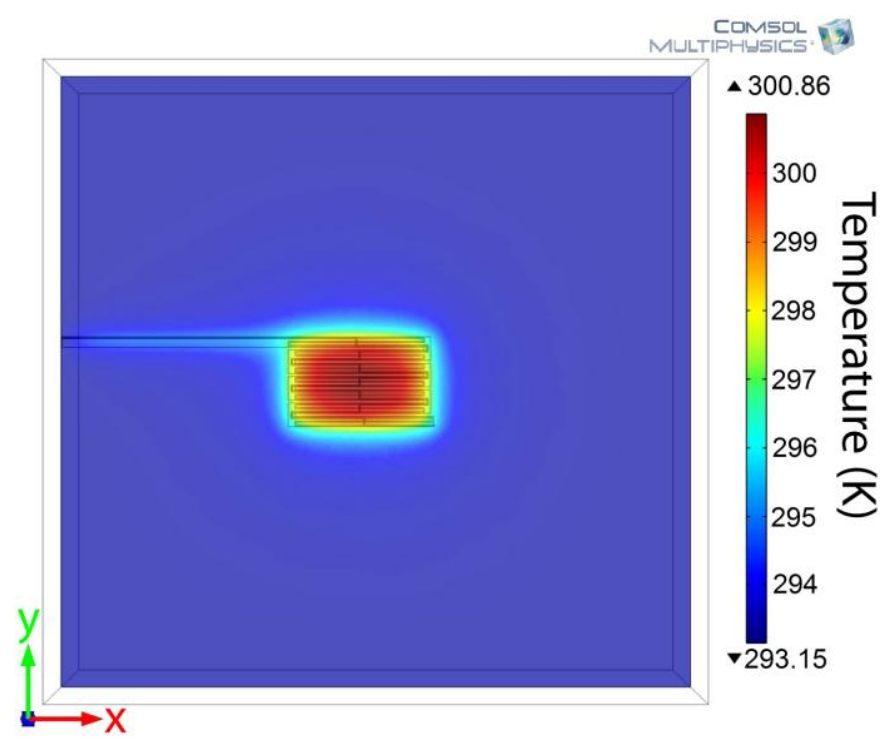

Fig. 15. Cross-sectional profile in $x y, 15 \mu \mathrm{m}$ below the actuator. The temperature elevation is at most $7.7 \mathrm{~K}$, indicating that its high thermal conductivity allows the $\mathrm{Si}$ substrate to efficiently remove the generated heat energy.

\section{Simulations}

A finite element model (FEM) was built in order to quantify the temperature elevation of the MEMS and its surroundings. The model comprised a single actuator spring, identical with the fabricated one, submerged into a volume of water and positioned $20 \mu \mathrm{m}$ above a Si substrate. The model was solved in Comsol 4.2a using the heat conduction and resistive heating physics modules. Material constants from the program library were used. The largest withstandable current, $6 \mathrm{~mA}$, was applied through the actuator electrode. The surrounding boundaries were set to $293.15 \mathrm{~K}$.

The results are shown in fig. 14 and fig. 15, the prior revealing that the electrode itself heats up by about $29 \mathrm{~K}$, which through analysis can be shown to give a displacement in the correct range. In the zoom-in in fig. 14, a comparison between the profile above and below the actuator shows that the $\mathrm{Si}$ substrate efficiently removes the heat energy generated by the MEMS.

Fig. 15 shows a cross-section in $x y, 15 \mu \mathrm{m}$ below the structure. At this level, directly below the actuator, the maximum temperature elevation is $7.7 \mathrm{~K}$. This result suggests that a "bio-friendly thermal window" can be found, giving sufficient displacement without harming the cells. The result also underlines that a tip height of 20-30 $\mu \mathrm{m}$ will help substantially to thermally decouple the sample from the MEMS.

\section{CONCLUSIONS}

We presented the design, fabrication and characterization of a novel device using an integrated long-range thermal actuator to displace a force sensing cantilever. The system was conceived for bio-AFM applications. The aim is to use such individually actuated devices in arrays to measure several samples in parallel and thereby increasing the through-put.
A first generation of devices has been successfully fabricated and characterized, reaching close to $17 \mu \mathrm{m}$ of displacement in air. Throughout this displacement, the optical reference structure (ORS) followed the platform in a linear and attenuated way. The change temperature was measured based on the change of resistivity and the relation between displacement and temperature change was found to be linear, as expected from theory. A thermomechanical sensitivity of $\sim 77.6 \mathrm{~nm} / \mathrm{K}$ was determined, which corresponds well with the results of our analytical estimation that gave a value of $\sim 68.8$ $\mathrm{nm} / \mathrm{K}$.

A novel usage of the RIE lag effect was presented, showing how it can be used to induce a controlled thinning of the $\mathrm{Si}$ where needed in order to improve optical access. The resulting etch depth can be controlled by varying the density and size of the openings in the hard-mask.

Simulations suggest that a local temperature elevation of some $29 \mathrm{~K}$ could be induced in the electrode layer while working in water. Given that a substrate of high thermal conductivity is selected, the temperature of the substrate has a dominating influence on the resulting temperature of the water layer closeby. This is crucial in order not to induce harmful temperatures at the level of the cell. Through a parylene deposition, the electrodes were passivated. A device was tested in water, confirming the functionality of this postprocessing step.

The measured thermomechanical sensitivity would result in about $2.2 \mu \mathrm{m}$ of deformation for a temperature change of 29 $\mathrm{K}$. Consequently, the next challenge is to achieve higher thermomechanical sensitivity in order to assure that a large displacement range is maintained in water.

\section{ACKNOWLEDGMENT}

The authors gratefully acknowledge the Nano-Tera Swiss federal program for supporting this work financially through the PATLiSci project. At CSEM, Neuchâtel, we also thank André Meister and Mélanie Favre for advice regarding the readout system, and Yves Petremand for his help in the dynamic MEMS characterization. We thank Shuangyi Zhao at the Ceramics Laboratory, EPFL, for his help in measuring the temperature coefficient of resistivity. We thank the staff at CMi-EPFL for their valuable support during the fabrication.

\section{REFERENCES}

[1] S. Suresh, "Biomechanics and biophysics of cancer cells," Acta Biomaterialia, vol. 3(4): p. 413-438 2007.

[2] B. Drake, C.B. Prater, A.L. Weisenhorn, S.A.C. Gould, T.R. Albrecht, C.F. Quate, D.S. Cannell, H.G. Hansma, and P.K. Hansma, "Imaging crystals, polymers, and processes in water with the atomic force microscopy," Science, vol. 243(4898): p. 1586-1589 1989.

[3] S. Husale, H.H.J. Persson, and O. Sahin, "DNA nanomechanics allows direct digital detection of complementary DNA and microRNA targets," Nature, vol. 462(7276): p. 1075-U138 2009.

[4] G.U. Lee, D.A. Kidwell, and R.J. Colton, "Sensing discrete streptavidin biotin interactions with atomic-force microscopy," Langmuir, vol. 10(2): p. 354-357 1994.

[5] R. Matzke, K. Jacobson, and M. Radmacher, "Direct, high-resolution measurement of furrow stiffening during division of adherent cells," Nature Cell Biology, vol. 3(6): p. 607-610 2001.

[6] H.G. Hansma, J. Vesenka, C. Siegerist, G. Kelderman, H. Morrett, R.L. 
This article has been accepted for publication in a future issue of this journal, but has not been fully edited. Content may change prior to final publication.

Henriksson, Gullo and Brugger: Long-range thermal actuators for parallelizable bio-AFM

Sinsheimer, V. Elings, C. Bustamante, and P.K. Hansma, "Reproducible imaging and dissection of plasmid DNA under liquid with the atomic force microscope," Science, vol. 256(5060): p. 1180-1184 1992.

[7] G. Binnig, C.F. Quate, and C. Gerber, "Atomic force microscope," Physical Review Letters, vol. 56(9): p. 930-933 1986.

[8] T.R. Albrecht, S. Akamine, T.E. Carver, and C.F. Quate, "Microfabrication of cantilever styli for the atomic force microscope," Journal of Vacuum Science \& Technology a-Vacuum Surfaces and Films, vol. 8(4): p. 3386-3396 1990.

[9] G.E. Fantner, R.J. Barbero, D.S. Gray, and A.M. Belcher, "Kinetics of antimicrobial peptide activity measured on individual bacterial cells using high-speed atomic force microscopy," Nature Nanotechnology, vol. 5(4): p. 280-285 2010.

[10] B. Lee, C.B. Prater, and A.P. King, "Lorentz force actuation of a heated atomic force microscope cantilever," Nanotechnology, vol. 23(5) 2012.

[11] M. Lekka, P. Laidler, D. Gil, J. Lekki, Z. Stachura, and A.Z. Hrynkiewicz, "Elasticity of normal and cancerous human bladder cells studied by scanning force microscopy," European Biophysics Journal with Biophysics Letters, vol. 28(4): p. 312-316 1999.

[12] S.E. Cross, Y.-S. Jin, J. Rao, and J.K. Gimzewski, "Nanomechanical analysis of cells from cancer patients," Nature Nanotechnology, vol. 2: p. 780-783 2007.

[13] S.C. Minne, G. Yaralioglu, S.R. Manalis, J.D. Adams, J. Zesch, A. Atalar, and C.F. Quate, "Automated parallel high-speed atomic force microscopy," Applied Physics Letters, vol. 72(18): p. 2340-2342 1998.

[14] P. Vettiger, G. Cross, M. Despont, U. Drechsler, U. Durig, B. Gotsmann, W. Haberle, M.A. Lantz, H.E. Rothuizen, R. Stutz, and G.K. Binnig, "The "millipede" - Nanotechnology entering data storage," Ieee Transactions on Nanotechnology, vol. 1(1): p. 39-55 2002.

[15] J. Haaheim, V. Val, J. Bussan, S. Rozhok, J.W. Jang, J. Fragala, and M. Nelson, "Self-Leveling Two-Dimensional Probe Arrays for Dip Pen Nanolithography (R)," Scanning, vol. 32(1): p. 49-59 2010.

[16] J. Polesel-Maris, L. Aeschimann, A. Meister, R. Ischer, E. Bernard, T. Akiyama, M. Giazzon, P. Niedermann, U. Staufer, R. Pugin, N.F.d. Rooij, P. Vettiger, and H. Heinzelmann, "Piezoresistive cantilever array for life sciences applications," Journal of Physics: Conference Series, vol. 61: p. 955-959 2007.

[17] M. Favre, J. Polesel-Maris, T. Overstolz, P. Niedermann, S. Dasen, G. Gruener, R. Ischer, P. Vettiger, M. Liley, H. Heinzelmann, and A. Meister, "Parallel AFM imaging and force spectroscopy using twodimensional probe arrays for applications in cell biology," Journal of Molecular Recognition, vol. 24(3): p. 446-452 2011.

[18] F. Loizeau, T. Akiyama, S. Gautsch, A. Meister, P. Vettiger, and N.F. de Rooij, "Two-dimensional cantilever array with varying spring constants and tip radii for life-science applications," Micro \& Nano Letters, vol. 7(4): p. 301-305 2012.

[19] T. Sulchek, R.J. Grow, G.G. Yaralioglu, S.C. Minne, C.F. Quate, S.R. Manalis, A. Kiraz, A. Aydine, and A. Atalar, "Parallel atomic force microscopy with optical interferometric detection," Applied Physics Letters, vol. 78(12): p. 1787-1789 2001.

[20] T. Sulchek, R. Hsieh, J.D. Adams, S.C. Minne, C.F. Quate, and D.M. Adderton, "High-speed atomic force microscopy in liquid," Review of Scientific Instruments, vol. 71(5): p. 2097-2099 2000.

[21] T. Akiyama, L. Aeschimann, L. Chantada, N.F. De Rood, H. Heinzelmann, H.P. Herzig, O. Manzardo, A. Meister, J. Polesel-Maris, R. Pugin, U. Staufer, and P. Vettiger, "Concept and demonstration of individual probe actuation in two-dimensional parallel atomic force microscope system," Japanese Journal of Applied Physics Part 1Regular Papers Brief Communications \& Review Papers, vol. 46(9B): p. 6458-6462 2007.

[22] G.E. Fantner, D.J. Burns, A.M. Belcher, I.W. Rangelow, and K. YoucefToumi, "DMCMN: In Depth Characterization and Control of AFM Cantilevers With Integrated Sensing and Actuation," Journal of Dynamic Systems Measurement and Control-Transactions of the Asme, vol. 131(6) 2009.

[23] S.H. Lim, J. Choi, R. Horowitz, and A. Majumdar, "Design and fabrication of a novel bimorph microoptomechanical sensor," Journal of Microelectromechanical Systems, vol. 14(4): p. 683-690 2005.

[24] K.M. Jia, S. Pal, and H.K. Xie, "An Electrothermal Tip-Tilt-Piston Micromirror Based on Folded Dual S-Shaped Bimorphs," Journal of Microelectromechanical Systems, vol. 18(5): p. 1004-1015 2009.

[25] J. Gere and S. P. Timoshenko, Mechanics of Materials, 4 ed. Boston, MA: PWS, 1997

[26] J.S. Pulskamp, A. Wickenden, R. Polcawich, B. Piekarski, M. Dubey and G. Smith, "Mitigation of residual film stress deformation in multilayer microelectromechanical systems cantilever devices," Journal of Vacuum Science and Technology B, vol. 21(6): p. 2482-2486 2003.

[27] H. Jansen, M. deBoer, R. Wiegerink, N. Tas, E. Smulders, C. Neagu, and M. Elwenspoek, "RIE lag in high aspect ratio trench etching of silicon," Microelectronic Engineering, vol. 35(1-4): p. 45-50 1997.

[28] C.K. Chung, "Geometrical pattern effect on silicon deep etching by an inductively coupled plasma system," Journal of Micromechanics and Microengineering, vol. 14(4): p. 656-662 2004.

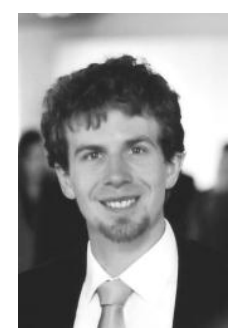

Jonas Henriksson received his MSE and MSc degrees in Applied Physics and Electrical Engineering from Linköping University, Sweden. $\mathrm{He}$ is currently pursuing a $\mathrm{PhD}$ degree in Microengineering at Ecole Polytechnique de Lausanne (EPFL), Switzerland. His current research interests include MEMS technologies, bio-sensing, gas sensing and microactuator systems.

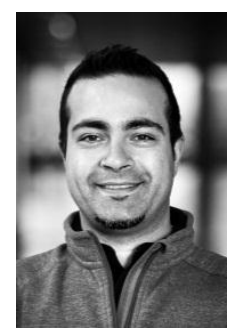

Maurizio R. Gullo is currently working as scientific collaborator at the institute of microtechnology at the Ecole Polytechnique Federale de Lausanne (EPFL), Switzerland. Formerly he was application manager for scanning probe microscopes (SPM) at Nanosurf AG in Switzerland. He received his MSc degree in Applied Physics and his $\mathrm{PhD}$ degree in Nanotechnology from Neuchatel University, Switzerland, where he successfully combined SPM and electrochemistry for in-situ electric current measurement on biological cell membranes.

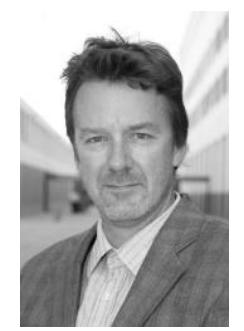

Juergen Brugger is Professor at EPFL in Microengineering and Materials Science and is active in the field of interdisciplinary micro- and nanotechnologies with a focus on novel manufacturing techniques for integrated and multi-functional micro/nanosystems. $\mathrm{He}$ is coauthor of $>160$ peer-reviewed scientific publications $(\mathrm{H}=28)$, is member of the editorial board of "Nanotechnology" from the Institute of Physics (IoP). Dr. Brugger is co-inventor of more than 15 patents and he has received three IBM patent awards. He served on the program committees of IEEE-IEDM, IEEE-MEMS, IEEE NEMS, Transducers, Eurosensors and MNE conferences, and is currently elected co-chair of IEEE-MEMS 2015. Juergen Brugger is senior member of IEEE, co-founder of the start-up MicroLeman Sarl and board member of the "Omega Award Foundation". 